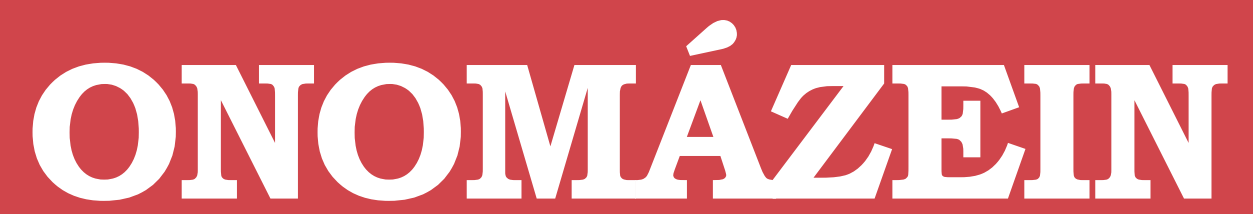

Revista semestral de lingüística, filología y traducción
PONTIFICIA UNIVERSIDAD CATÓLICA DE CHILE FACULTAD DE LETRAS

\title{
Tense in a vectorial model for the conceptualization of time ${ }^{1}$
}

\author{
Ismael Iván Teomiro García \\ Universidad Nacional de Educación a Distancia \\ España
}

María Beatriz Pérez Cabello de Alba

Universidad Nacional de Educación a Distancia

España

\section{(C) $\underset{\mathrm{BY}}{(\mathrm{i})}$}

Ismael Teomiro: Departamento de Filologías Extranjeras y sus Lingüísticas, Facultad de Filología, Universidad Nacional de Educación a Distancia, España. | Correo electrónico: iteomiro@flog.uned.es

M. Beatriz Pérez: Departamento de Filologías Extranjeras y sus Lingüísticas, Facultad de Filología, Universidad Nacional de Educación a Distancia, España. | Correo electrónico: bperez-cabello@flog.uned.es 


\section{Abstract}

In this work we assume that the human mind cannot perceive time directly and thus resorts to a metaphor of space in order to conceptualize it (Casasanto \& Boroditsky, 2008; Gentner et al., 2002; Merrit et al., 2010). We argue that time, which is conceptualized in terms of a path image schema, is needed along with spatial coordinates in order to locate a proposition in a possible world so that it can receive a truth-value. In other words, both time and space are needed to evaluate a proposition. The human mind codifies the temporal properties of a proposition by means of three systems, which are based upon Reichenbach's (1947) temporal variables, namely speech time, evaluation time and utterance time: tense, which locates an event or situation along the temporal path image schema (past, present or future); aspect, which represents the speaker's viewpoint of the event or situation conveyed in the utterance (perfectivity and progressivity, among others); and lexical aspect or aktionsart, which encodes the temporal properties of the event or situation itself (i.e. whether it is bound, unbound, or punctual). Specifically, we provide a mathematical model that represents the information codified by these three systems by means of a Euclidean vector (a geometric entity characterized by a magnitude, which in our case is a number times an abstract temporal unit) in a four-dimensional-like mental representation, namely an $\mathrm{R}^{3}+\hat{t}$ mental representation: a three dimensional space $\left(R^{3}\right)$ defined by three versors (a vector whose magnitude equals one unit and defines a line), $\hat{x}, \hat{y}$ and $\hat{z}$ plus a fourth versor $\hat{t}$ that defines the temporal path image schema along which the proposition must be placed in order to receive a truth value. Ultimately, this work aims to offer a novel account of tense using theoretical tools from cognitive linguistics and formal logic, as well as mathematical formalisms, which will allow us to carry out the computational implementation of the model in NLP systems.

Keywords: tense; time; vector; idealized cognitive model; image schema.

1 This work has been partially funded by research projects FFI2011-29798-C02-01 (Spanish Ministry of Economy and Competitiveness) and 2013-014-UNED-PROY (Spanish National University of Distance Education). 


\section{Introduction}

This work sets out to address a number of questions related to the conceptualization of time from a new perspective. First, we will tackle the issue of how the human mind conceptualizes time. Our goal is to contribute to this debate by putting into question whether time is an inherent property of the world external to the individual and if not, why the human mind needs the concept of time and what for. We will briefly review the works of Reichenbach (1947) about tense and aspect, and Vendler (1967) regarding lexical aspect.

Second, we will propose a model for the conceptualization of time and space rooted in the framework of Cognitive Linguistics. More precisely, we will start from the embodied cognition thesis in Linguistics based on works by Lakoff (1987, 1993), and Johnson (1987), among others, as well as the Conceptual Metaphor Theory (Lakoff, 1993; Lakoff \& Johnson, 1980), and pursue the already discussed idea that time is conceptualized by means of a more concrete domain of experience like space (Casasanto \& Boroditsky, 2008; Gentner et al., 2002; Merrit et al., 2010; Weger \& Pratt, 2008). From this idea about the metaphorical understanding of time as space, we will follow the Conceptual Metaphor Theory in that the same conceptual devices used to conceptualize space are used to conceptualize time (Casasanto \& Boroditsky, 2008; Gentner et al., 2002; Merrit et al., 2010). Thus, we will propose that space is conceptualized as a three dimensional mental representation defined by three path image schemata. The novelty of this work is that a fourth path image schema is proposed to define a line that will be used to encode the temporal properties of states of affairs.

Third, we will use mathematical tools in order to formalize the aforementioned model based on path image schemata. We will express the temporal-spatial mental model in mathematical terms as an $\mathrm{R}^{3}$ space that requires a $t$ line, i.e. an $\mathrm{R}^{3}+\hat{t}$. Both the spatial axes and the temporal line will be defined by means of Euclidean versors, and the states of affairs as Euclidean vectors.

Finally, we will formalize the expression of the temporal properties of states of affairs in this $\mathrm{R}^{3}+\hat{t}$ mental representation using mathematical formalisms like the aforementioned vectors, and from Set Theory (Cantor, 1874). This is a task that will not be completely fulfilled in this work due to space reasons. Therefore, we will focus on tense, leaving aside lexical aspect (aktionsart) and aspect for other works (Pérez Cabello de Alba \& Teomiro García, in progress; Teomiro García \& Pérez Cabello de Alba, in progress). This mathematical formalization, mainly based on Euclidean vectors, is extremely useful for this model to be used in natural language processing, as we will discuss in the final section.

\section{Theoretical background}

\subsection{A brief note on the ontological sta- tus of time}

The debate on how the human mind conceptualizes time dates back to Classical Greece. Time has been a central topic in the history of philosophy ever since Aristotle's concepts of "te-

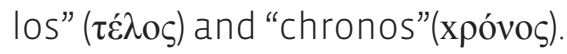

It seems clear that both space and time are needed in order to understand the natural world or the external reality, as it will be called in this work hereinafter. Aristotle considered time as part of the natural world and, as such, part of the discipline of physics. However, new trends in physics point towards the epiphenomenal nature of time in a similar way as it occurs with other concepts like causation, as Reinhart (2000) argues:

We should note, however, that despite the formal appearance of ( $\mathrm{lb}$ ), this is not a formal logical formula. 
(1) a. Max broke the glass.

b. Lexical semantics (based on Chierchia, 2004)2: $\lambda x \lambda y \exists b$ [CAUSE (b)y, BROKEN (x)

(Paraphrase: some action $b$ of $y$ caused $x$ be[come] broken)

c. Max broke the glass $=>$ The glass broke.

As is well known, CAUSE is not a logical relation. The only definable entailment that can be associated with the CAUSE relation is precedence: if CAUSE holds between an action $b$ and an instance $i$ of the property $\lambda x(\operatorname{BROKEN}(X))$, then $b$ precedes i (and by standard inference, i holds, since b\&i holds). This should be sufficient to guarantee the entailment in (IC), which is indeed crucial. But no further truth conditions (entailments) can be associated with (1a)-E.g. we cannot logically infer from ( $\mathrm{Ib}$ ) that if not $\mathrm{b}$, then not BROKEN $(\mathrm{x})$ (Reinhart, 2000: 38).

Thus, Reinhart claims that causation is not a logical property inherent to the external reality but rather a device that our conceptual system uses in order to apprehend the reality and give coherence to it. As for time, let us take movement as an example of a phenomenon requiring the concept of time in order to be apprehended. If an element $A$ is perceived in a given position $\alpha$ and in another position $\beta$ as in figure 1 , then the mind has to resort to the concept of movement3: A has moved from $\alpha$ to $\beta$, and so, it can be at both places (but not at the same time), as represented in figure 2.

Movement requires a series of temporally ordered stages. In other words, movement involves time. However, quantum physics has shown that an element, under certain circumstances, can be at two places at once (O'Connell et al., 2010). As a result, there is no need for movement nor time in order to explain the displacement of elements if this reasoning is on the right track (at least not in all the cases of this phenomenon). It is not our goal to discuss whether or not time is an inherent

\section{FIGURE 1}

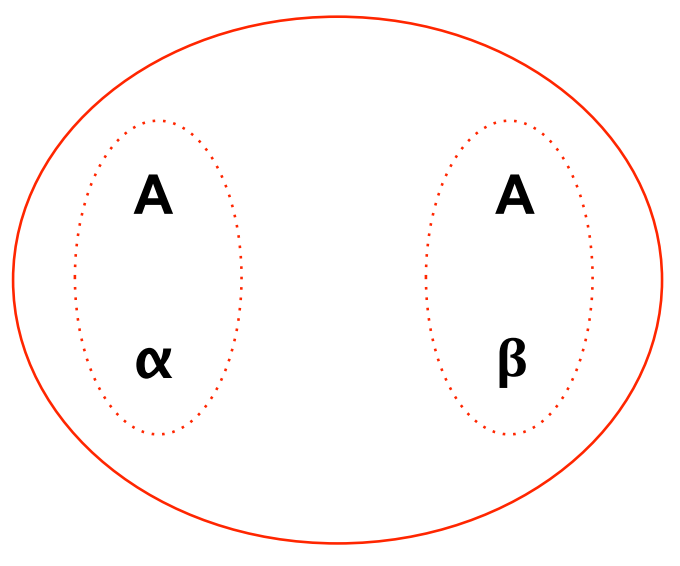

FIGURE 2

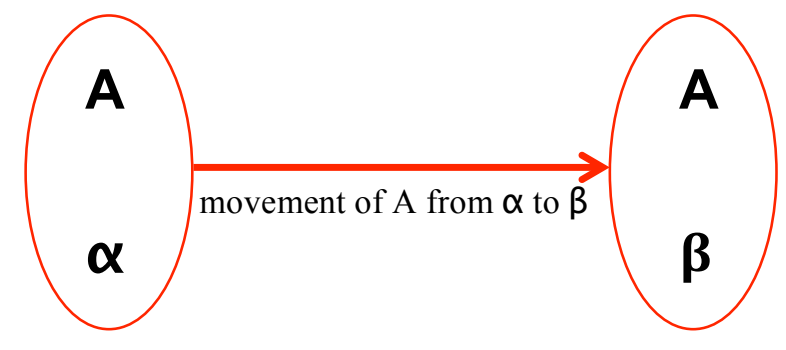

property of the external reality but just to point out the possibility of time being a device used by the mind in order to apprehend the external reality, perhaps in a similar way as causation (Choi, 2009; Dainton, 2010; Lucas, 1973; Reichenbach, 1958; Reichenbach, 1991; Whitrow, 1980).

\subsection{Tense, aspect, and aktionsart}

Traditionally, three temporal properties of states of affairs have been distinguished: tense, aspect and lexical aspect or aktionsart (Taylor, 1977; Bach, 1981; Bach, 1986; Bennett \& Partee, 1972; Burges, 1984; Comrie, 1985; Dahl, 1985; Giorgi \& Pianesi, 1997; Heny, 1982; Prior, 1967; Vendler, 1967; Verkuyl, 1972; Verkuyl, 1993, among others).

Tense serves the function of placing a state of affairs in time with respect to the speaker's time.

2 The original citation is Chierchia (1989), which was published later in 2004.

3 It seems clear that movement undeniably happens in external reality but this might not hold for all situations, as shown by O'Connell et al. (2010). 
It distinguishes past for states of affairs previous to the speaker's time as in (2), future for states of affairs following the speaker's time as in (3), and present for states of affairs partially or completely overlapping with the speaker's time as in (4):

(2) a. John ate the pizza.

b. John had eaten the pizza.

(3) a. John will eat the pizza.

b. John will have eaten the pizza.

(4) a. John is eating the pizza right now.

b. The moon moves around the Earth.

Aspect, on the other hand, constrains the viewpoint or perspective of a state of affairs by presenting it as finished (perfective aspect) as in (5), unfinished (imperfective) as in (6), or being repeated over time as in (7):

(5) John has built the house.

(It implies that the house was built and finished in reference to a given point in time)

(6) John was building the house.

(It implies that the house was not built yet in reference to a given point in time)

(7) John was coughing during the meeting.

(It implies that John coughed a number of times during the meeting)

Finally, lexical aspect or aktionsart describes the internal temporal properties of the state of affairs. For example, whether it is dynamic (i.e. an event like [8]) or static (i.e. a state like [9]); or whether it is punctual as in (10) or has duration as in (11), among other properties.

(8) The tree fell.

(9) John loves Mary.

(10) The bomb exploded (*for three hours).

(11) Mary read the book in three hours.

\subsection{Reichenbach (1947): Tense and as- pect in English}

Probably the work by the philosopher Reichenbach (1947) has provided one of the most illuminating insights about time ever. A section of his 1947 book revolves around tense, aspect and their interaction in English. He defined time as a device to determine the position of states of affairs with reference to the temporal point of the act of speech, i.e. the moment in which the speaker utters the speech act. In order to formalize time, he developed a linear system where time was defined as a line along which a state of affairs is placed in a point he called event time. The act of speech (i.e. the temporal point where the speaker utters the state of affairs) can be also placed along that temporal line in a point he called speech time. Finally, the temporal point $\mathrm{R}$, called reference time, is the one that corresponds to the viewpoint or psychological vantage point adopted by the speaker.

According to Reichenbach, the relations among the three temporal points (event, speech and reference) can define all the tenses of the English language. For example, in the sentence "John is eating the pizza" in (12), the reference, event and speech time coincide and so is interpreted as present tense (i.e. the event is going on at the same time it is being uttered). Another example is the sentence "John ate the pizza" in (13), where the reference and event time coincide but both precede the speech time. This yields a past tense interpretation: the state of affairs took place before the moment in which the speaker utters the sentence that conveys it.

(12) John is eating the pizza.

(13) John ate the pizza.

\subsection{Vendler (1967): Aktionsart in modern linguistics}

With regard to aktionsart, it is the work of Vendler (1967) that brought these features, previously identified by Aristotle, to modern linguistics (Borer, 2005; Borik, 2002; Borik \& Reinhart, 2004; Demirdache \& Uribe-Etxebarria, 2000; Hornstein, 1990; Krifka, 1998; Levin, 2000; Van Valin, 2005; Vendler, 1967; Verkuyl, 1972; Verkuyl, 1993, among many others). Vendler studied the temporal properties of the states of affairs and 
identified four basic features of the temporal structure of states of affairs:

- Telicity is the property of a predicate, usually a verb, that conveys a state of affairs being complete, i.e. with an end point.

- Punctuality is the property of a predicate that conveys a state of affairs that takes place in a minimal abstract unit of time. If a state of affairs is punctual, then it has no duration (or its duration equals the minimal abstract unit of time).

- Duration is the extension of time in which the state of affairs takes place.

- Dynamicity is the property of a predicate that conveys a state of affairs that somehow changes through time.

On the basis of these features, he defined four basic types of states of affairs:

- States are static and atelic (i.e. do not have an end point), like the predicates in (14).

(14) a. I love cakes.

b. I know all your secrets.

c. I don't feel well at present.

- Activities are dynamic and atelic, as in (15).

(15) a. John built houses.

b. I run in the weekends.

c. I ate lots of cakes when I was younger

- Accomplishments are dynamic and telic (i.e. have an end point), like the examples in (16).

(16) a. John built the house.

b. I read the book in two hours.

c. I ate three cakes last week.

- Achievements are telic and punctual, like the predicates in (17).

(17) a. I noticed that there was something missing.

b. I sneezed a lot when I caught a cold last week.

c. After hours, I finally figured it out.
From the seminal paper by Vendler many other works have provided a deeper understanding of aktionsart, and refined the taxonomy of states of affairs (see references above). This brief review cannot do justice to the extensive work on this issue, and our intention is just to give a basic overview of how these temporal properties of states of affairs came to the foreground in modern linguistics.

\subsection{Time in cognitive semantics}

We draw on cognitive linguistics and pursue two of its basic guidelines: the embodied cognition thesis (conceptual structure is embodied; Lakoff \& Johnson, 1999; Evans \& Green, 2006), and that semantic structure is conceptual structure (Lakoff \& Johnson, 1999; Evans \& Green, 2006; Johnson, 1987; Lakoff, 1987; Lakoff, 1993). We thus assume that concepts and meaning are embodied, and that our conceptualization of the external reality is done through idealized cognitive models, more specifically through image schemata (Johnson, 1987), as well as conceptual projection (Johnson, 1987; Lakoff, 1987; Lakoff, 1993) and cross-domain mappings as stated in the Conceptual Metaphor Theory (Lakoff, 1993; Lakoff \& Johnson, 1980).

Johnson (1987) argued that embodied experience gives rise to image schemata within the conceptual system. Image schemata are abstract conceptual representations derived from sensory and perceptual experience as we interact with and move about in the world. For example, since humans walk upright, and we have a head at the top of our bodies and feet at the bottom, and given the presence of gravity which attracts unsupported objects, the vertical axis of the human body is functionally asymmetrical. Consequently, the vertical axis is characterized by an up-down or top-bottom asymmetry: the top and bottom parts of our bodies are different. The UPDOWN image schema is derived from this aspect of our experience. 
The human conceptual system is composed of idealized cognitive models (ICMs), which are relatively stable mental representations of the reality formed through a process of idealization. They are idealised because they abstract across a range of experiences rather than representing specific instances of a given experience. They guide cognitive processes like categorization and reasoning (Lakoff, 1987).

Image schemata are ICMs that serve as the foundation for conceptual structure. Lakoff (1987) argues that our experience and concepts of SPACE are structured in large part by image schemata like CONTAINER, SOURCE-PATH-GOAL, PART-WHOLE, UP-DOWN, FRONT-BACK and SO On.

According to Lakoff \& Johnson (1980), conceptual metaphors give rise to systems of conventional conceptual mappings, held in longterm memory, which may be motivated by image-schematic structure. Image schemata arise from bodily experience, and hence conceptual metaphor consists in mapping rich and detailed structure from concrete domains of experience (usually image schemata) onto more abstract concepts and conceptual domains. For example, the conceptual domain Love is understood through a metaphorical mapping to the conceptual domain JOURNEY. In other words, the conceptual metaphor Love is a JOURNEY allows us to understand the more abstract domain Love through several links to the more concrete domain JOURNEY:

- The lovers (LOVE) are travellers (JOURNEY).

- The relationship (LOVE) is a path (JOURNEY), PATH image schema).

- The problems in the relationship (LOVE) are obstacles (JOURney).

- Marriage, cohabitation and offspring (LOVE) are the destiny (JOURNEY).
It is widely known that in cognitive linguistics time is conceptualized by means of the metaphor TIME is SPACE. Thus, time is a concept that is understood my means of a metaphorical extension of space (Choi, 2009; Brala Vukanović \& Gruić Grmuša, 2009; Casasanto \& Boroditsky, 2008; Gentner et al., 2002; Merrit et al., 2010; Sinha \& Bernárdez, 2015). In other words, the conceptual metaphor TIME is SPACE allows us to conceptualize TIME by means of SPACE. This can be seen in many linguistic expressions as those in (18), many of which make use of spatial prepositions in order to refer to time:

(18)a. He was looking at me from the beginning to the end.

b. Shall we meet at 10 o'clock?

c. I'll get there in an hour.

d. I ran to get to the exam on time but I finaIly arrived late.

e. You still have ten minutes left to finish your exams. At 12 o'clock you will be out of time.

Besides, the field of psycholinguistics provides us with evidence that indicates that in many languages time is perceived as a linear function, i.e. in terms of a horizontal linear axis (Wearden \& Jones, 2007; Weger \& Pratt, 2008) ${ }^{4}$.

\section{The conceptualization of SPACE in a 3-dimensional mental representation}

We assume as our null hypothesis that every proposition needs to be located both in time and space with respect to a possible world in order to be interpreted. In other words, every proposition requires temporal and spatial coordinates in order to be properly understood by the semantic systems. We believe, though, that the proposition needs to be located within a mental representation of time and space referring to a

4 However, the fact that time is mentally represented along a horizontal line depends on cultural factors, as shown by Sinha \& Bernárdez (2015). 
possible world rather than in temporal-spatial coordinates necessarily belonging to the external reality. As a matter of fact, the conceptual and linguistic systems do not compute the coordinates themselves but some relations that are established in the mental representation defined by such coordinates (see section 6).

Putting time aside till next section, a mental representation of a three-dimensional space needs three axes: $x, y$, and $z$, as represented in figure 3 below.

\section{FIGURE 3}

Three-dimensional space representation

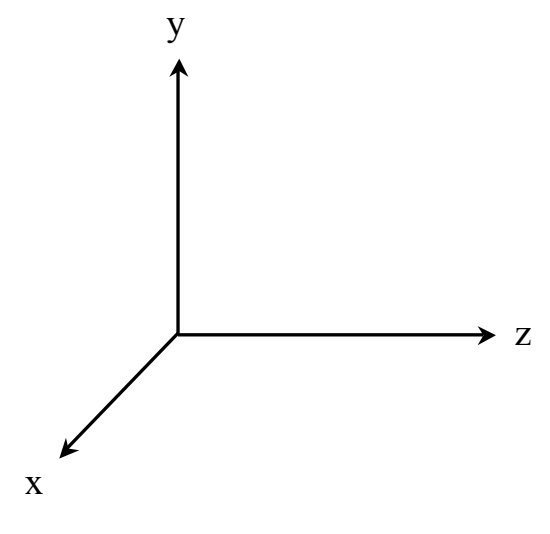

Our hypothesis is that this three-dimensional mental representation is made up of three path image schemata. According to Johnson (1987: 115), a path schema, represented in figure 4 , is an image schema that involves physical or metaphorical movement from place to place and consists of a starting point, a goal, and a series of intermediate points 5 .

\section{FIGURE 4}

Path image schema

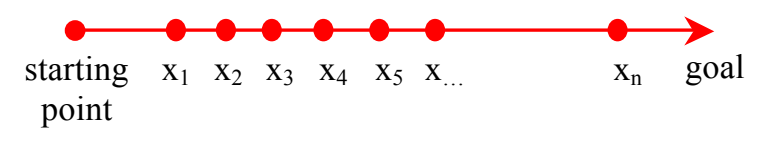

In order to define an axis, we only need a line. In turn, the only elements that are required to define a line are a starting point and a goal as long as they differ from each other. This requires that the path image schema has (at least) one intermediate point different from both the starting point and the goal. We will call this case of path image schema minimal path image sche$\mathrm{ma}$, which is represented in figure $5^{6}$.

\section{FIGURE 5}

Minimal path image schema

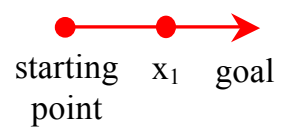

starting point $\neq$ goal

$\mathrm{x} 1 \neq$ starting point

$\mathrm{x} 1 \neq$ goal

Thus, in order to define the three axes $(x, y$, and $z$ ) of our three-dimensional mental representation we need three minimal path image schemata that define a line each. Let us call these minimal path image schemata $x^{\text {m.p.i.s. }}, y^{\text {m.p.i.s. }}$ and $z^{\text {m.p.i.s. }}$ Our three-dimensional spatial mental representation can be graphically represented as below.

\section{FIGURE 6}

Three-dimensional mental representation defined by minimal path image schemata

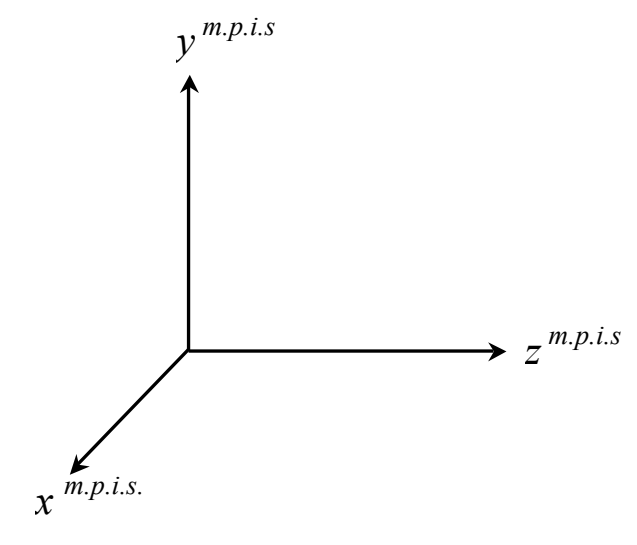

5 Path image schemata will be formalized by means of Euclidean vectors, as we will argue in section 5.

6 Minimal path image schemata will be formalized by means of Euclidean versors, as we will argue in section 5 . 


\section{TIME is SPACE: The conceptualization of time in a 4-like dimensional mental representation}

In this section we are going to argue that the conceptual devices used to conceptualize space (idealized cognitive models and more precisely path image schemata) are used to conceptualize time too by means of cross-domain mappings (i.e. the conceptual metaphor time is SPACE).

Our proposal is that time is also conceptualized by means of a minimal path image schema,

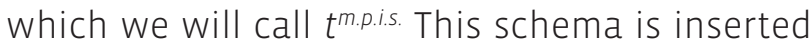
in the three dimensional mental representation (see previous section) and defines a temporal line, which will be called $t$, as represented in figure 7.

\section{FIGURE 7}

Temporal-spatial mental representation defined by minimal path image schemata

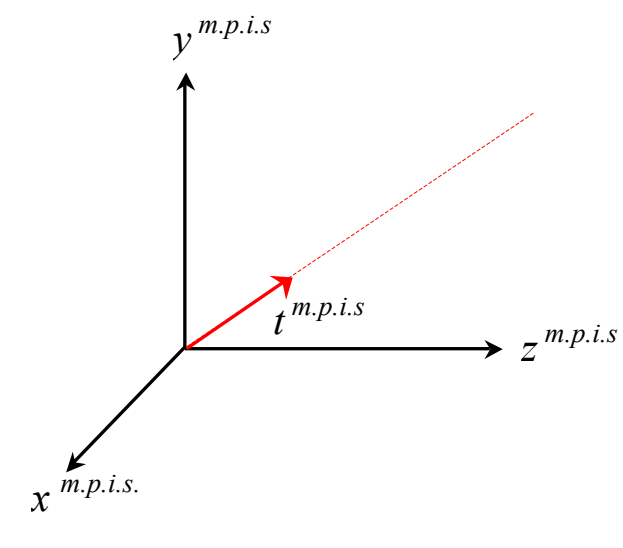

Note that the line defined by the temporal minimal path image schema does not contribute a fourth axis to the mental representation. This remains a three-dimensional representation of space plus an additional line that encodes the temporal properties of states of affairs. The spatial properties are encoded by means of the three spatial axes ( $x, y$, and $z)$. Therefore, we will not call this mental representation a four dimensional space but a four-like dimensional one: it remains three-dimensional whilst it requires an additional line that acts as a supplementary axis.
Now we can reformulate the null hypothesis introduced in the previous section by saying that every proposition needs to be located in a four-like temporal-spatial mental representation with respect to a possible world in order to be interpreted. This amounts to saying that every proposition needs to be placed, at the conceptual level (i.e. in a mental representation), both in a series of spatial coordinates and along a temporal line in order to be understood.

\section{The formalization of the temporal- spatial mental representation: $R^{3}+\hat{t}$ and Euclidean vectors}

We believe that the path image schemata that define the temporal-spatial mental representation can be formalized by means of mathematical devices. Thus we propose to use Euclidean vectors to formalize path image schemata. This facilitates the representation of certain relationships established among them within the temporal-spatial mental representation. The advantage is that this formalization will allow us to represent temporal properties in a very simple way, while it will be easy to implement it in natural language processing systems, as we will discuss in the last section.

As mentioned above, we propose Euclidean vectors as a means to formalize path image schemata. Note that the whole temporal-spatial mental representation is built from path image schemata, so the whole mental representation can be expressed in terms of Euclidean vectors. The term Euclidian indicates that these vectors have properties different from the vectors defined in mathematics or physics. Hereinafter we will assume that all vectors are Euclidean and then omit this term.

A vector is a geometric entity having magnitude and direction that connects an initial point A with a terminal point $B$, as shown in figure 8 .

A vector is denoted as $\overrightarrow{A B}$ and is characterized by: 


\section{FIGURE 8}

Euclidean vector

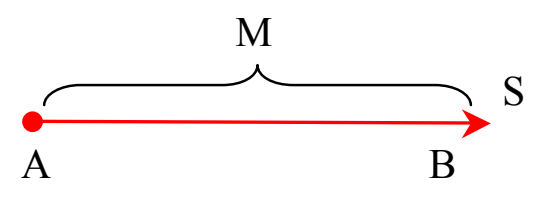

- An initial point $A$, represented as $[A(\overrightarrow{A B})]$.

- A terminal point $B$, represented as $[B(\overrightarrow{A B})]$.

- A magnitude $M$, represented as $[M(\overrightarrow{A B})]$, which is a number times an abstract unit. In our case, an abstract spatial unit if the schema defines a spatial axis, or an abstract temporal unit if the schema defines the temporal line. The entire quantity of abstract units is split into the orthogonal-axis components of the vector.

- A direction, defined by the segment formed between the initial point $[A(\overrightarrow{A B})]$ and the terminal point $[B(\overrightarrow{A B})]$.

- And a sense, represented as $[S(\overrightarrow{A B})]$, which is positive if the initial point is $[A(\overrightarrow{A B})]$ and the terminal point is $[B(\overrightarrow{A B})]$, or negative if it is defined the other way around.

We propose to represent path image schemata by means of a vector:

- The initial point of the path image schema is the initial point $[A(\overrightarrow{A B})]$ of the vector.

- The terminal point of the path image schema is the terminal point $[B(\overrightarrow{A B})]$ of the vector.

- Like a path image schema, the vector $\overrightarrow{A B}$ has intermediate points. The number of intermediate points is the magnitude $[M(\overrightarrow{A B})]$ of the vector.

A versor, represented as $\widehat{A B}$, is a special kind of vector. It has the same elements as a vector. Its distinguishing property is that its magnitude is one, i.e. $[M(\widehat{A B})]=1$. Recall that we argued in the previous section that the spatial path schemata defining the spatial axis only needed one intermediate point because that is enough in order to define a line. We called this kind of path image schemata minimal path image schemata. Versors, rather than vectors, can represent this kind of minimal schemata because only one intermediate point is necessary to define a line. This amounts to saying that the vector representing these schemata need have a magnitude $[M(\overrightarrow{A B})]=1$, which is the minimal to define a line. This means that our spatial three-dimensional mental representation can be said to be formed out of three versors: $\hat{x}, \hat{y}$ and $\hat{z}$. In mathematical terms, this is an $\mathrm{R}^{3}$ space, represented in figure 9.

\section{FIGURE 9}

An $R^{3}$ space

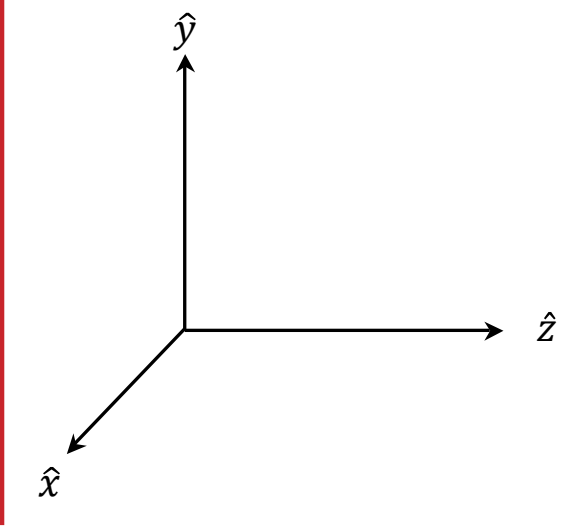

The temporal line is also defined in terms of a minimal path image schema. Therefore it can be formalized in terms of a versor too. So we can define our temporal-spatial four-like dimensional representation as an $\mathrm{R}^{3}+\hat{t}$ mathematical space whose axes are defined by three versors $(\hat{x}, \hat{y}$ and $\hat{z}$ ), and a temporal line is formed by a temporal versor $(\hat{t})$, as represented in figure 10 .

Finally, note that any point in an $\mathrm{R}^{3}$ space has a corresponding position vector. If we define a point $\alpha$ in an $\mathrm{R}^{3}$ space, then a corresponding position vector can be defined by determining its initial point in the zero point of the three axes, i.e. $[A(\vec{\alpha})]=X_{0}, Y_{0}, Z_{0}$, and its terminal point in $\alpha$, i.e. $[B(\vec{\alpha})]=X_{\alpha} Y_{\alpha} Z_{\alpha}$. This is represented in figure 11 .

With these theoretical tools, we can now define Reichenbach's temporal points in our $\mathrm{R}^{3}+\hat{t}$ space. 


\section{FIGURE 10}

An $R^{3}+\hat{t}$ space

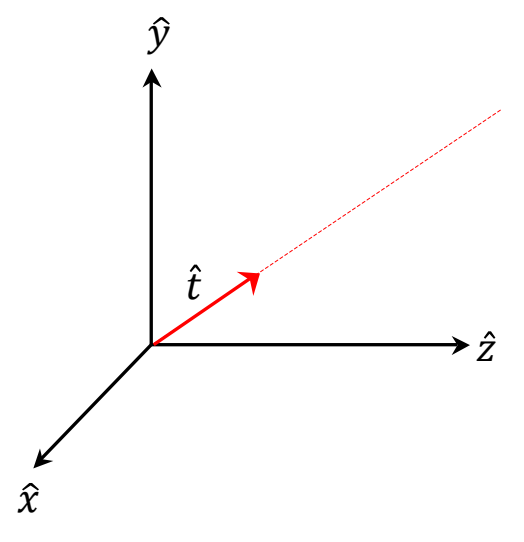

- Speech time (S): can be defined as a point situated along the temporal line defined by $\hat{t}$. Since any point in an $\mathrm{R}^{3}$ has a corresponding position vector, an $\hat{S}$ position vector can be defined as that whose initial point is the zero point in the three spatial axes, i.e. $[A(\vec{S})]=X_{0} Y_{0} Z_{0}+T_{0}$, and its terminal point is $S$, i.e. $[A(\vec{S})]=X_{s}+Y_{s}+Z_{s}+T_{s}$.

- Reference time (R): can be defined as a vector $\vec{R}$. We will not develop on the nature of this vector in this work for the sake of simplicity?

\section{FIGURE 11}

The position vector $\vec{\alpha}$ corresponding to the point $\alpha$
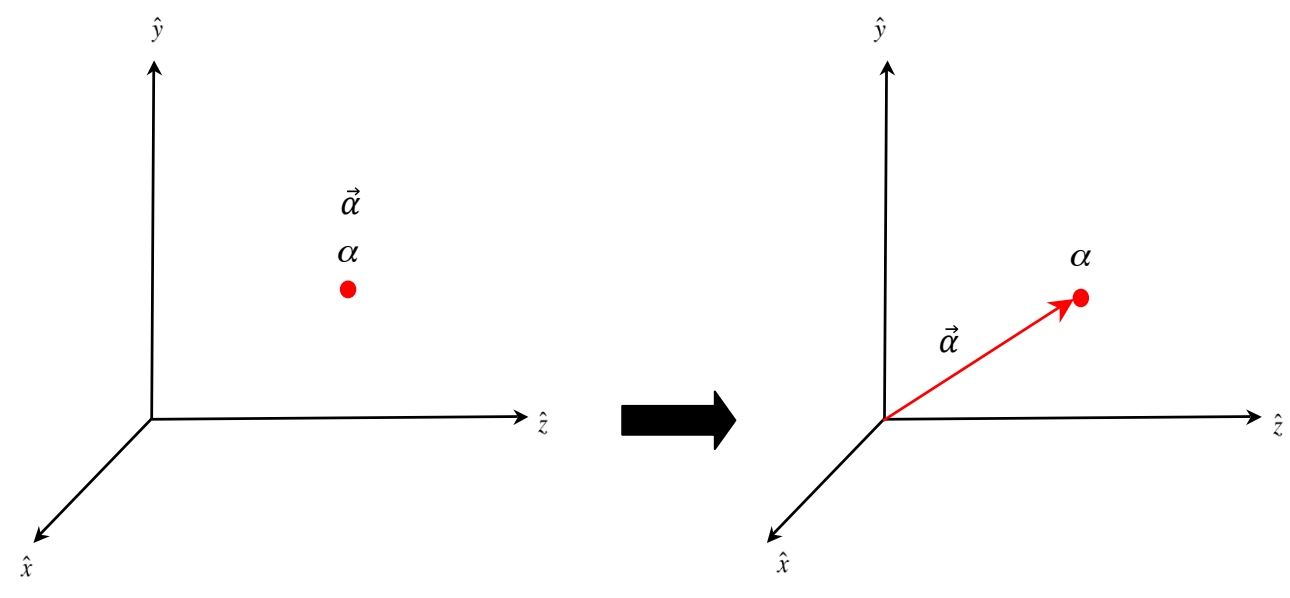

- Event time (E): depending on the internal temporal properties of the state of affairs, it can be a line, a segment or a vector $\vec{E}$. Again, we have to leave this for future work and assume, for the sake of simplicity, that the event time is a vector $\vec{E}$ whose initial point $[A(\vec{E})]$ is the beginning of the state of affairs and its terminal point $[B(\vec{E})]$ is the end of the state of affairs ${ }^{8}$.

The three vectors $(\vec{S}, \vec{R}$, and $\vec{E})$ are situated within a three-dimensional mental representation $\left(R^{3}\right)$ and along a temporal line $(t)$. In other words, these three vectors are located in an $\mathrm{R}^{3}+\hat{t}$ space, where the temporal properties of the state of affairs conveyed by the proposition are encoded.

Back to our initial hypothesis, it can be revisited so as to state that every proposition needs to be located in an $\mathrm{R}^{3}+\hat{t}$ temporal-spatial mental representation in order to be properly processed by the semantic systems. In other words, every state of affairs conveyed by a proposition needs to be located at the conceptual level, along a temporal line within a three-dimensional space. Note that this is in line with Wearden \& Jones

7 See Teomiro \& Pérez Cabello de Alba (in progress).

8 See Pérez Cabello de Alba \& Teomiro (in progress). 


\section{FIGURE 12}

A state of affairs within an $\mathrm{R}^{3}+\hat{t}$ representation

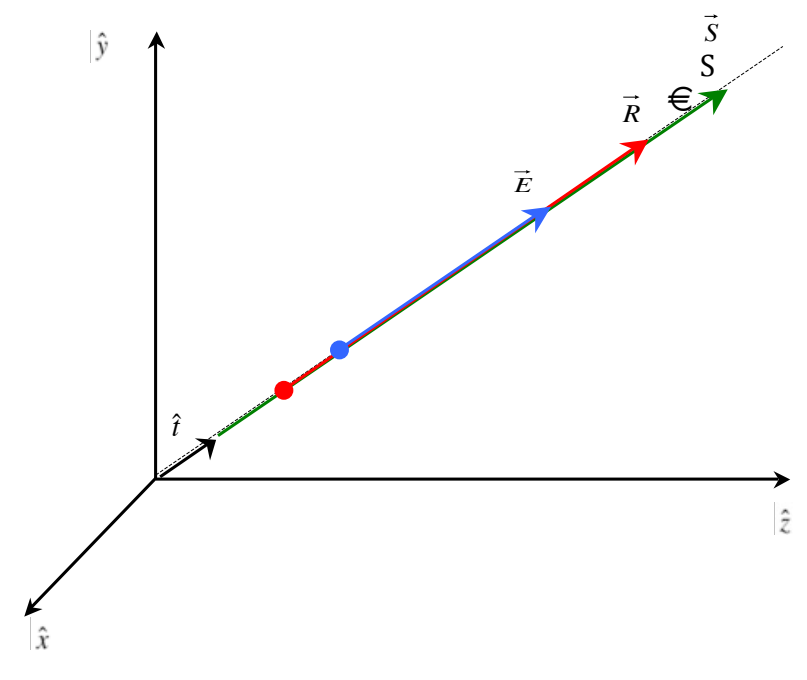

(2007) as well as with Weger \& Pratt (2008), who defend that the perception of time is a linear function?.

We will see below that the absolute values of the points and vectors are not crucial for the conceptual system or the linguistic system. These systems only reflect certain relations among the $\vec{S}, \vec{R}$, and $\vec{E}$ vectors, which will render the temporal properties of the states of affairs (see next section).

\section{The conceptualization of tense}

As mentioned above, in this work we focus on one of the three temporal systems seen in section 2.2: the tense system. We follow Borik (2002) in the assumption that tense is encoded by means of relations between $\mathrm{E}$ (event time) and $\mathrm{S}$ (speech time). Other relations like the one between $\mathrm{S}$ and $\mathrm{R}$ (reference time), and between $\mathrm{R}$ and $\mathrm{E}$ encode a different temporal system, namely the aspect system (Teomiro \& Pérez Cabello de Alba, in progress). The internal temporal properties of the state of the affairs will be encoded by means of certain properties of the vector re- presenting $\mathrm{E}$ in our $\mathrm{R}^{3}+\hat{t}$ representation (Pérez Cabello de Alba \& Teomiro, in progress).

We now move on to formalize deictic tense on the temporal-spatial $\mathrm{R}^{3}+\hat{t}$ mental representation by means of the relations established between the $\vec{E}$ and $\vec{S}$ vectors. We assume that the tense system only differentiates three tenses at most: present, past, and future.

\subsection{Present}

We start by discussing present tense. As we argued in section 2.2, a state of affairs is in present tense if it takes place (E) at the same time as the time the speaker utters the speech act (S). However, this tense does not distinguish whether the event begins to take place before the S time or at the same time as S. All the examples in (19) are in the present tense although in (19a) the event is understood to have begun a bit earlier than S, in (19b) the event is understood to have begun a lot earlier than $\mathrm{S}$, and in (19c) the event is understood to have begun roughly at the same time as S. As we see in these examples, other linguistic devices are needed if we want to distinguish this kind of nuance: aspect (continuous vs. simple), periphrasis (be just, starting to), etc. Obviously, the event cannot begin after the $\mathrm{S}$ time since the latter is a point (it has no duration) and then they could not be said to take place at the same point.

(19) a. I'm eating the pizza.

b. The Earth moves around the sun.

c. I'm just starting to read the book you lent me.

In our $\mathrm{R}^{3}+\hat{t}$ mental representation, this tense can be defined by resorting to two relations (from set theory) between the vectors $\vec{E}$ and $\vec{S}$, namely, intersection and inclusion. A state of affairs that is in the present tense is taking place at the same time as the speech time. However, 
nothing is implied about its starting or ending points but only that the state of affairs cannot be finished before the point of speech. We can formalize this by saying that two conditions have to be met for a state of affairs to be in the present tense:

- First, the intersection between the vectors $\vec{E}$ and $\vec{S}$ is different from zero. This can be formalized as follows: $\vec{E} \cap \vec{S} \neq 0$ (intersection).

- Second, the vector $\vec{S}$ does not contain the vector $\vec{E}$. The formalization of this is: $\vec{E} \notin \vec{S}$ (inclusion).

Present time can thus be graphically represented as below (only the temporal line is drawn for the sake of simplicity) (Figure 13)

\subsection{Past}

In section 2.2 we argued that a state of affairs is in the past tense when the event time (E) was before the speech time (S) in the temporal line, regardless of other factors like aspect or lexical aspect, as can be seen in (20) and (21) respectively.

(20)a. I was eating the pizza.

(Progressive aspect) b. All moved when the earthquake took place. (Simple aspect)

c. I had read the book you lent me. (Perfective aspect)

(21)a. I built the house.

(Telic)

b. I built houses.

(Atelic)

c. I loved John.

(Non-dynamic)

d. I found the keys.

(Punctual)

The past tense can be defined in our $\mathrm{R}^{3}+\hat{t}$ mental representation by resorting to the intersection and inclusion relations between the vectors $\vec{E}$ and $\vec{S}$. A state of affairs that takes place in the past tense has started and has finished before the point of speech. So two conditions have to be met in order for a state of affairs to be in the past tense:

First, that the intersection between the vectors $\vec{E}$ and $\vec{S}$ is different from zero. This is denoted as follows: $\vec{E} \cap \vec{S} \neq 0$ (intersection).

Second, that the vector $\vec{S}$ contains the vector $\vec{E}$. The formalization of this is: $\vec{E} \notin \vec{S}$ (inclusion)

\section{FIGURE 13}

Present tense

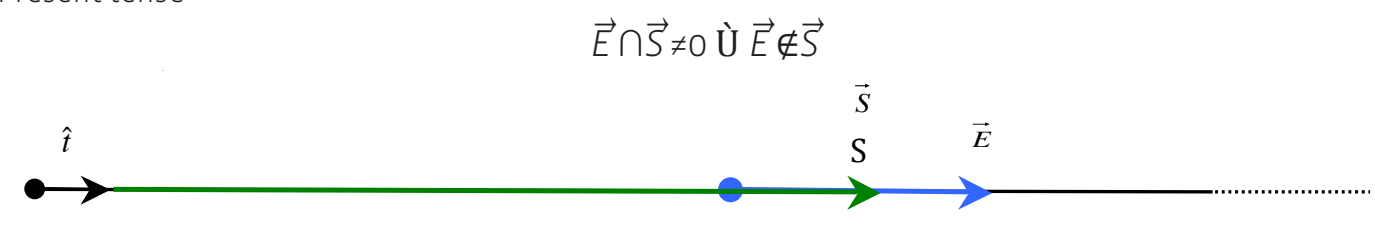

FIGURE 14

Present tense

$\vec{E} \cap \vec{S} \neq 0$ Ù $\vec{E} \notin \vec{S}$

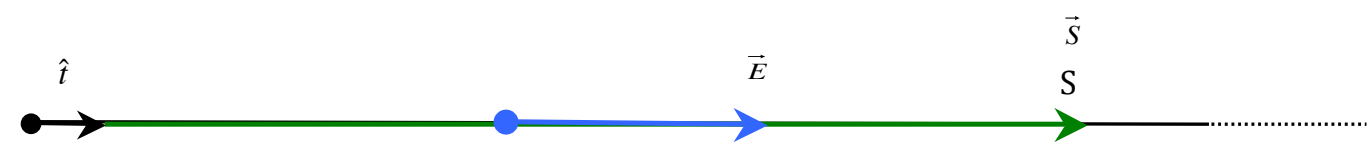




\subsection{Future}

A state of affairs is in the future tense if it has not happened by the time of speech. In other words, the event time $\mathrm{E}$ is located after the speech point $S$ in the temporal line regardless other factors like aspect and lexical aspect, as can be seen in (22) and (23) respectively.

(22)a. I will be eating the pizza.

(Progressive aspect)

b. All will move when the earthquake takes place.

(Simple aspect)

c. I will have read the book you lent me. (Perfective aspect)

(23)a. I will build the house. (Telic)

b. I will build houses. (Atelic)

C. I will love John. (Non-dynamic)

d. I will find the keys. (Punctual)

The future tense can be formalized in our $\mathrm{R}^{3}+\hat{t}$ mental representation by resorting to the inclusion relation between the vector $\vec{E}$ and $\vec{S}$. A state of affairs that takes place in the future has not started before or at the same time as the speech time, nor has it finished before or at the same time as the speech time. Therefore, the condition that has to be met for a state of affairs to be in the future tense is only one:

That the intersection between the vectors $\vec{E}$ and $\vec{S}$ equals zero. This is denoted as follows: $\vec{E} \cap \vec{S}=0$ (intersection).

\section{Conclusions and implications for fu- ture research}

To conclude, we have provided a unified model for the conceptualization of time, graphically represented below. Here, we have focused on tense due to space reasons but we are working on the systems of aspect and lexical aspect $(\text { aktionsart })^{10}$. This model is based on idealized cognitive models, and, more specifically, on path image schemata. These schemata have, in turn, been mathematically formalized by means of Euclidean vectors.

By using mathematical formalisms, the three tenses can be defined in terms of intersection and inclusion conditions:

\section{FIGURE 15}

Future tense

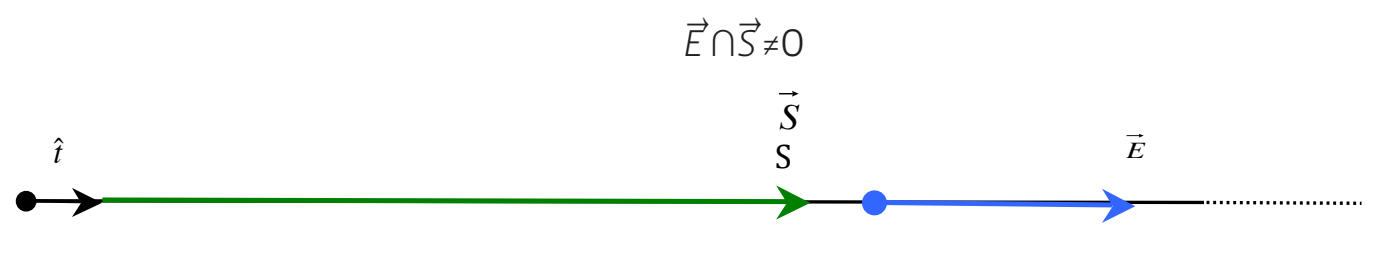

10 The extension of this model for the aktionsart and aspect systems will be done in further work (Pérez Cabello de Alba \& Teomiro, in progress; Teomiro \& Pérez Cabello de Alba, in progress). The aspect system will be developed by formalizing diverse relations established between the vectors $\vec{S}$ and $\vec{R}$, and $\vec{E}$ and $\vec{R}$ (following Borik \& Reinhart, 2004). The formalization of the lexical aspect or aktionsart will be done by means of properties of the vector $\vec{E}$, which in some cases will be a vector, a line or a segment. The telicity of the event will be explained by resorting to the nature of the initial and terminal points of the event $\vec{E}$. Also, the interaction among tense, aspect and aktionsart will be formalized in the model we propose in future work. For example, continuous tenses in English yield an unfinished reading of the state of affairs. However, if the state of affairs is a semelfactive (Smith, 1991), then an iterative reading emerges. We will provide mathematical tools that capture this and similar phenomena. 


\section{FIGURE 16}

A state of affairs within an $\mathrm{R}^{3}+\hat{t}$ representation

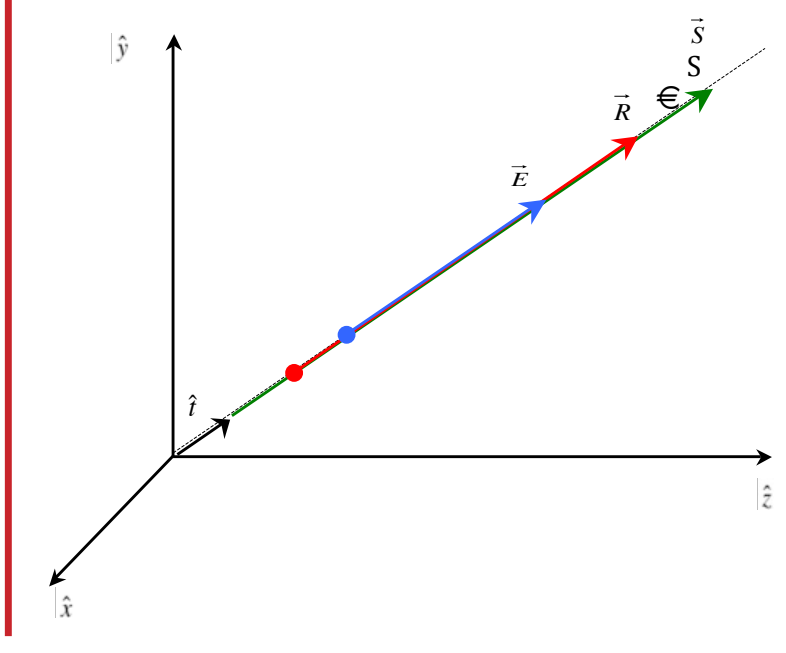

(24)Tense: $\vec{E}$ - $\vec{S}$ relations
a. Present: $\vec{E} \cap \vec{S} \neq 0 \wedge \vec{E} \notin \vec{S}$
b. Past: $\quad \vec{E} \cap \vec{S} \neq 0 \wedge \vec{E} \notin \vec{S}$
c. Future: $\vec{E} \cap \vec{S}=0$

This analysis can be applied to the study of the conceptual-linguistic interface and the grammaticalization of time across languages. For example, in Germanic languages the verbal morphology can only encode past or present, but not future. This is because the verbal morphology only encodes the inclusion relation between $\vec{E}$ and $\vec{S}$. A modal (will) is needed to encode a non-default intersection between $\vec{E}$ and $\vec{S}$. On the contrary, in Romance languages the verbal morphology encodes past, present, and future. This is because the verbal morphology encodes both inclusion and intersection between $\vec{E}$ and $\vec{S}$. We see that, at the conceptual level, the elements that define tense in both families of languages are the same, but the way in which each family decides to grammaticalize them is different.

Finally, the model we have presented in this paper can help improve the representation of the temporal properties of an utterance through an interlingua, which can be used in natural lan- guage processing. For example, this model could be used for enriching the tense operators used in ARTEMIS (Automatic Representation of TExt Meaning via an Interligua-based System; Periñán Pascual \& Arcas Túnez, 2014). This parser will build the semantic representation of a text where the temporal properties of the sentences are encoded by means of tense operators. However, there are some temporal properties that cannot be encoded by means of those operators. For example, in a multilingual knowledge base such as FunGramkB (Periñán Pascual \& Arcas Túnez, 2010), the difference between the "pretérito" (preterite) (25) and the "pretérito imperfecto" (imperfect preterite) (26) in Spanish cannot be represented by ARTEMIS because the tense operators are insufficient. We aim to represent these temporal nuances by means of the tools provided by the proposed model.
(25)Anduvo $\quad 5 \mathrm{~km}$ todos los días. Walked $_{\text {perfecto-35G }} 5 \mathrm{~km}$ all the days "He walked $5 \mathrm{~km}$ every day."
(26)Andaba $\quad 5 \mathrm{~km}$ todos los días. Walked $_{\text {imperfecto-35G }} 5 \mathrm{~km}$ all the days "He walked $5 \mathrm{~km}$ every day."
(27)“...peroayer no llegó a 4.” ... but yesterday not came ${ }_{35 G}$ to 4
"... but yesterday he couldn't do even $4(\mathrm{~km})$."

As seen in above, both (25) and (26) can be translated as "He walked $5 \mathrm{~km}$ every day". However, we find differences in the logical implications of (25) vs. (26): A speaker can say (26) followed by (27) without provoking infelicity. However, if a speaker says (25) followed by (27), the felicity conditions are not met. In order to properly represent this difference, English needs to resort to a modal as in (28) or to a periphrasis as in (29). So "He walked 5 km every day" is a proper translation of (25), whereas (28) and (29) are more accurate translations of (26).
(28)He would walk $5 \mathrm{~km}$ every day.
(29)He used to walk 5 km every day. 
The difference encoded by the different types of "pretérito" in Spanish (kinds of past tense) is encoded by means of a modal or verbal periphrasis in English. This difference can be represented in our model through the properties of the temporal vectors $\vec{E}, \vec{S}$, and $\vec{R}$, and using set theory terminology:

(30)Spanish "Pretérito Simple" (cf. 25) and English Past Simple: Past $(\vec{E} \cap \vec{S} \neq 0 \wedge \vec{E} \notin \vec{S}) \wedge \vec{R} \neg \notin \vec{E}$

(31)Spanish "Pretérito imperfecto" (cf. 26) and English "would" and "used to": Past $(\vec{E} \cap \vec{S} \neq 0 \wedge \vec{E} \notin \vec{S}) \wedge \vec{R} \notin \vec{E}$

What we can see formalized in (30) is that the event is in the past tense and that the speaker evaluates it after it is finished (the vector $\vec{R}$ is not included within the vector $\vec{E}$ ). Hence, the event is perceived as complete. On the other hand, the formalization in (31) involves a past event that is evaluated before it is finished (the vector $\vec{R}$ is included within the vector $\vec{E}$ ), which gives a nonfinished reading ${ }^{11}$.

Another example of different codification of temporal properties across languages is the case of the English adverb "just" and the Spanish verbal periphrasis "acabar de" (have just done something). In the English sentence (32), the ad- verb "just" expresses that the event has finished at the same time as the speaker utters the act of speech. If we want to translate this sentence into Spanish, we would have to resort to the verbal periphrasis "acabar de" instead of an adverb, as in example (33).

(32) I've just arrived.
(33) Acabo de llegar.
Finish $_{\text {present-1SG }}$ of come $_{\text {infinitive }}$
"I've just arrived."

We can codify the semantic contribution of these two elements with the theoretical tools provided in the proposed model by using a relation of TERMINAL COINCIDENCE between the vector $\vec{E}$ and $\vec{S}$, which implies that the end of the vector $\vec{E}$ coincides with the beginning of the vector $\vec{S}$. So, ARTEMIs has to "know" that both the adverb "just" in English and the verbal periphrasis in Spanish are linked to this representation. This property cannot be represented with the existing operators in that system, so we propose to enrich them by means of attribute value matrixes that codify the different temporal properties that can be encoded in the model we have proposed, as in example (34) below:

(34)Atribute Value Matrix of the temporal properties of "I've just arrived" / "Acabo de llegar":

\section{'TENSE' $=\vec{E} \cap \vec{S} \neq 0 \wedge \vec{E} \in \vec{S}$ 'PRESENT'}

'AKTIONSART' = see Pérez Cabello de Alba \& Teomiro (in progress)

'ASPECT' = see Teomiro \& Pérez Cabello de Alba (in progress)

'OTHERS' $=\vec{E} \wedge \vec{S}=$ TERMINAL COINCIDENCE

11 The reader is referred to Teomiro \& Pérez Cabello de Alba (in progress) for a more detailed work on the codification of this kind of temporal property. 
To conclude, the model we have introduced in this paper can help natural language processing systems codify the temporal properties of sentences in a more appropriate and accurate way by providing them with new operators that relate to a four-like dimensional temporal-spatial representation. Its advantage rests on the fact that it accounts for the nuances of the tense paradigm in different languages, as we have exemplified with the past perfect in English and Spanish. Finally, our study brings together theoretical tools from cognitive linguistics and formal logic, as well as mathematical formalisms, which can be successfully used in a complementary way for explanatory purposes and computational implementation in NLP.

\section{References}

BACH, E., 1981: "On time, tense and aspect: An essay in English metaphysics" in R. Cole (ed.): Radical Pragmatics, New York: Academic Press, BACH, E., 1986: "The algebra of events", Linguistics and Philosophy 9, 5-16.

Bennett, M. and B. H. Partee, 1972: Towars the Logic of Tense and Aspect in English, Santa Monica, Ca: System Development Corporation.

Borer, Hagit, 2005: The Normal Course of Events, Oxford: Oxford University Press.

Borik, Olga, 2002: Aspect and Reference Time, Utrecht: LOT.

Borik, Olga and Tanya ReInhart, 2004: "Telicity and Perfectivity: Two Independent Systems", Proceedings of LOLA 8 (Symposium on Logic and Language), 13-34.

Brala Vukanović, M. and L. Gruić Grmuša (eds.), 2009: Space and Time in Language and Literature, Newcastle upon Tyne:Cambridge Scholars Publishing.

Burges, J. P., 1984: "Basic tense logic" in D. Gabbay and F. Guenthner (eds.): Handbook of Philosophical Logic Vol. 2, Dordrecht: Reidel.
Cantor, Georg, 1874: "Ueber eine Eigenschaft des Inbegriffes aller reellen algebraischen Zahlen", Journal für die reine und angewandte Mathematik 77, 258-62.

Casasanto, Daniel and Lera Boroditsky, 2008: "Time in the mind: Using space to thinkg about time", Cognition 106, 579-93.

Chierchia, Gennaro, 2004: "A Semantics for Unaccusatives and its Syntactic Consequences" in Artemis Alexiadou, Elena anagnostopoulou, and Martin Everaert (eds.): The Unaccusativity Puzzle, Oxford: Oxford University Press, 22-59.

Chol, S., 2009: "Typological differences in syntactic expressions of Path and Causation" in V. GathercoLE (ed.): Routes to Language: Studies in Honor of melissa Bowerman, Mahwah, JN: Lawrence Erlbaum Associates, 169-94.

Comrie, B., 1985: Tense, Cambridge: Cambridge University Press.

DAHL, Ö., 1985: Tense and Aspect Systems, Oxford: Blackwell.

Dainton, B., 2010: Time and Space, Second Edition, McGill-Queens University Press.

Demirdache, Hamida and Myriam Uribe-EtXebarria, 2000: "The Primitives of Temporal Relations" in Roger Martin, David Michaels, and Juan Uriagereka (eds.): Step by Step: Essays on Minimalist Syntax in Honor of Howard Lasnik, Cambridge, MA: MIT Press, 157-86.

Evans, Vyvyan and Melanie Green, 2006: Cognitive Linguistics. An Introduction, Chippenham, Wilys (UK): Edinburgh University Press.

Gentner, Dedre, Mutsumi Imal, and Lera Boroditsky, 2002: "As time boes by: Evidence for two system in processing space -> time metaphors", Language and Cognitive Processes 17, (5), 537-65.

GIorgl, Alessandra and Fabio PIANesI, 1997: Tense and Aspect, Oxford: Oxford University Press. 
Heny, F., 1982: "Tense, aspect and time adverbials. Part II", Linguistics and Philosophy 5, 109-54.

Hornstein, Norbert, 1990: As Times Goes By. Tense and Universal Grammar, MIT Press.

Johnson, Mark, 1987: The body in the mind: The bodily basis of meaning, imagination, and reason, Chicago: University of Chicago Press.

KRIFKA, Manfred, 1998: "The Origins of Telicity" in S. RothSTEIN (ed.): Events and Grammar, Dordrecht: Kluwer, 197-235.

LAKOFF, George and Mark JoHnson, 1980: "The metaphorical structure of the human conceptual system", Cognitive Science 4, 195-208.

LAKOFF, George, 1987: Women, fire and dangerous things: What categories reveal about the mind, Chicago: University of Chicago Press.

LakofF, George, 1993: "The Contemporary Theory of Metaphor" in Andrew Ortony (ed.): Metaphor and Thouht (Second Edition), Cambridge: Cambridge University Press, 202-51.

Lakoff, George and Mark Johnson, 1999: Philosohy in the Flesh: The Embodied Mind and its Challenge to Western Thought, New York: Basic Books.

Levin, Beth, 2000: Aspect, lexical semantic representation and argument expression, Proceedings of the 26th Annual Meeting of the Berkely Linguistics Society, 413-29

LUCAS, J., 1973: A Treatise on Time and Space, London: Methuen.

Merrit, Dustin J., Daniel Casasanto, and Elizabeth M. Brannon, 2010: "Do monkeys think in metaphors? Representations of space and time in monkeys and humans", Cognition 117, 191-202.

O’Connell, A. D. et al., 2010: "Quantum ground state and single-phonon control of a mechanical resonator", Nature 464, 697-704.
Pérez Cabello de Alba, María Beatriz and Ismael Iván TeOmiro García, in progress: Lexical aspect in a vectorial model for the conceptualization of time, unpublished manuscript, Universidad Nacional de Educación a Distancia.

Periñan Pascual, Carlos and Francisco Arcas Túnez, 2010: The architecture of FunGramKB, 7th International Conference on Language Resources and Evaluation, 2667-74, European Language Resources Association (ELRA).

Periñan Pascual, Carlos and Francisco Arcas Túnez, 2014: "The implementation of the FunGramkB CLS Constructor" in Brian Nolan and Carlos Perlñan Pascual (eds.): Language Processing and Grammars: The Role of Functionally Oriented Computational Models, Amsterdam/Philadelphia: John Benjamins, 165-96.

PrIOR, A., 1967: Past, Present and Future, Oxford: Oxford University Press.

Reichenbach, H., 1947: Elements of Symbolic Logic, New York: MacMillan.

Reichenbach, H., 1958: The Philosophy of Space and Time, Dover.

Reichenbach, H., 1991: The Direction of Time, University of California Press.

ReInHART, Tanya, 2000: "The Theta System: Syntactic Realization of Verbal Concepts", OTS Working Papers in Linguistics $(00,01 / \mathrm{TL})$,

Sinha, Chris and Enrique Bernardez, 2015: "Space, time and space-time. Metaphors, maps and fusions" in F. Sharifian (ed.): The Routledge Handbook of Language and Culture, New York: Routledge, 309-24.

Sмıтн, Carlotta S., 1991: The parameter of aspect, Dordrecht: Kluwer.

TAyLoR, B., 1977: "Tense and continuity", Linguistics and Philosophy 1, 199-220.

Teomiro García, Ismael Iván and María Beatriz Pérez Cabello de Alba, in progress: Aspect in a vectorial 
model for the conceptualization of time, unpublished manuscript, Universidad Nacional de Educación a Distancia.

Van Valin, Robert D. Jr., 2005: Exploring the syntaxsemantics interface, Cambridge: Cambridge University Press.

Vendler, Zeno, 1967: Linguistics in Philosophy, Ithaca: Cornell University Press.

VerkuyL, H. J., 1972: On the Compositional Nature of the Aspects, Dordrecht: Reidel.

VerkuyL, H. J., 1993: A theory of Aspectuality. The Interaction between Temporal and Atemporal Structures, Cambridge: Cambridge University Press.

Wearden, J. H. and Lukes A. Jones, 2007: "Is the Growth of Subjective Time in Humans a Linear or Nonlinear Function of Real Time?", The Quarterly Journal of Experimental Psychology 60, (9), 1289302.

Weger, U. W. and J. Pratt, 2008: "Time flies like an arrow: Space-time compatibility effects suggest the use of a mental time-line", Psychonomic Bulletin \& Review 15, 426-30.

Whitrow, G. J., 1980: The Natural Philosophy of Time, Oxford: Clarendon Press. 